\title{
Real-Time Simulation of an Aircraft Electric Driven Environmental Control System for Virtual Testing Purposes
}

\author{
Dirk Zimmer ${ }^{1}$, Niels Weber ${ }^{1}$, Peter Eschenbacher ${ }^{1}$ \\ ${ }^{1}$ DLR German Aerospace Center, Insitute of System Dynamics and Control, \\ Münchener Strasse 20, 82234 Oberpfaffenhofen, Germany, \\ dirk.zimmer@dlr.de
}

\begin{abstract}
A new object-oriented approach for the robust modeling of thermofluid systems has revealed to be also favorable for hard real-time simulation. This paper presents a complex and challenging application: an electric driven environmental control system architecture for potential use in future aircraft. The system is challenging to simulate in real-time because it also contains a computational expensive vapor cycle model and reconfigures because of switching bypasses. The system is complex to control due to its high degree of integration and interdependencies. Goal of this work is to integrate the model of this system for the virtual testing into an overall energy management for the aircraft on-board systems.
\end{abstract}

Keywords:Thermal fluids, Thermal process systems, real-time simulation, environmental control systems

\section{Motivation and Background}

\subsection{Virtual testing of an Energy Management Algorithm}

Algorithms for energy management are often a key design factor for the overall system design. This statements also holds true for our research field which is the system design of future More-Electric (or even All-Electric) Aircraft (MEA or AEA respectively). An intelligent energy management optimizes the use of power from different sources and thereby increases overall system efficiency. More important for an aircraft: the energy management can reduce peak loads and thereby help to reduce the weight of components such as generators or cables. This weight reduction in turn increases overall aircraft efficiency.

However, aircraft applications are subject to high safety standards and hence an energy management algorithm also has to be robust against failures or degradation of components. It is therefore necessary to test the interaction of the energy management algorithm with the corresponding subsystems.

A part of this testing has to be performed already at an early design stage when the systems are not yet available. Hence virtual testing is the only choice: here the energy management algorithm is executed on micro-controller architectures and the system response is generated by real-time simulation on conventional desktop PCs.

Especially interesting and challenging is the interaction of the energy management algorithm and the air generation unit (denoted as pack) of the environmental control system. It is interesting because the pack is the second largest power consumer on board only (vastly) surpassed by propulsion.

For the modelling and simulation community, this is challenging because neither the real-time simulation of such a pack nor the internal (prototypical) control are trivial tasks.

To gain a better understanding, let us first look at a Modelica model diagram of a potential electrical pack architecture and then study how this pack can interact with the energy management.

\subsection{An Electric-Driven Environmental Control System Architecture}

Figure 1 depicts a potential architecture for an electric vapour cycle pack (EVCP). It is one possible variant of the pack presented in (Golle, 2016), (Zimmer, 2018) and (Bender 2018). To gain a better understanding, let us remind that the function of a pack is to:

- provide a sufficient flow of fresh air (at different altitudes) to pressurize the cabin and keep air quality high.

- reach a desired pack discharge temperature (at different environmental conditions) to heat or cool the cabin.

- prevent fogging by ensuring an upper limit of the water content of the air.

We can now see these tasks being realized by the depicted architecture in the following way:

- Two compressors, a main compressor (5) and a smaller base compressor (3) provide the power for airflow generation, based on outside air (1). The base compressor can be bypassed (2) when not needed (as in operation near ground level). The primary heat-exchanger (4) between these two compressors prevents a too high outlet temperature of the main compressor. 
Real-Time Simulation of an Aircraft Electric Driven Environmental Control System for Virtual Testing Purposes

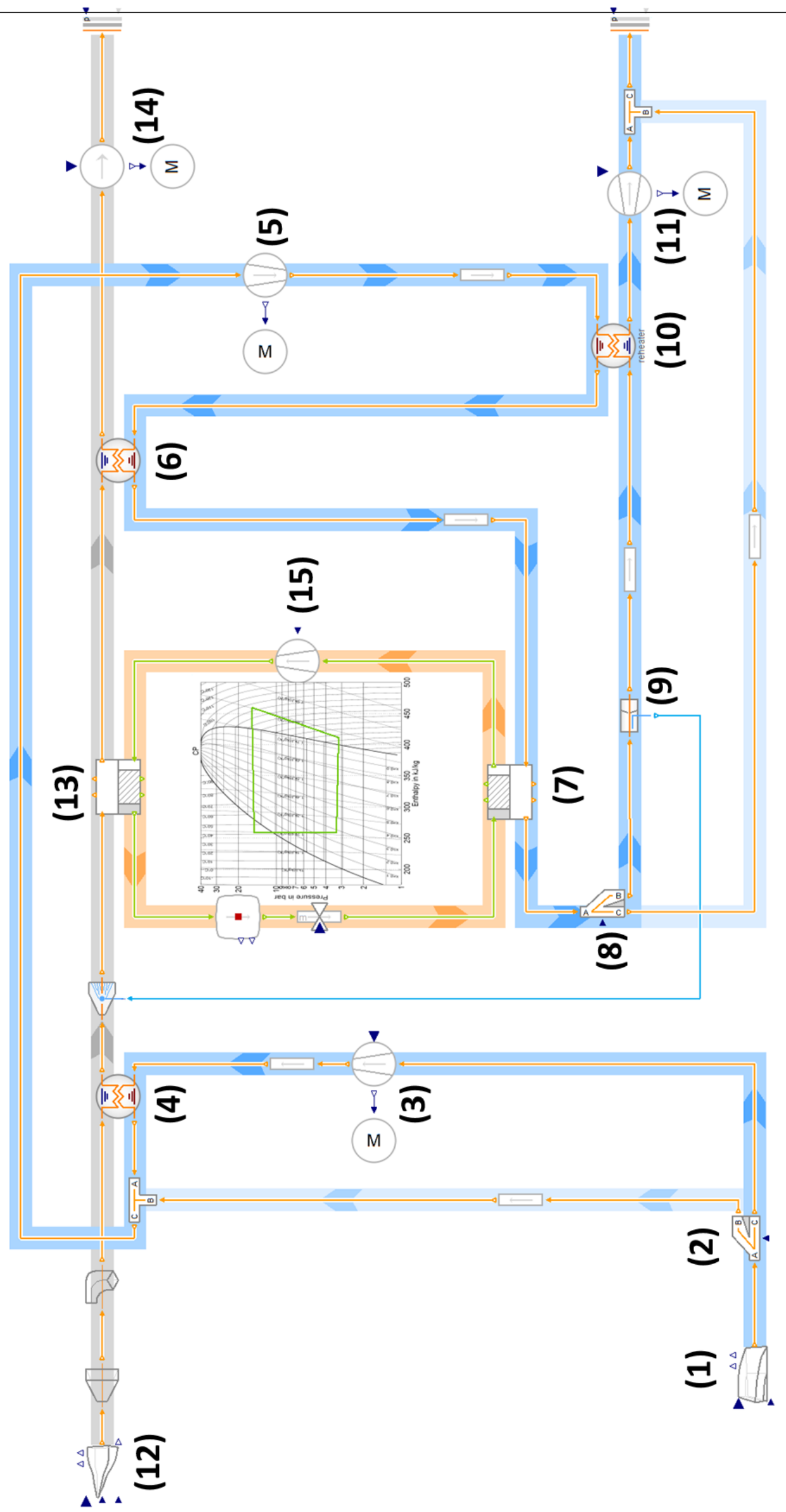

Figure 1: Modelica Diagram of an electric vapour cycle pack architecture (cleaned-up and freed from the control aspects) 
- Cooling of the air is achieved by using the main heat exchanger (6) and the condenser (13) and evaporator (7) of the electric driven (15) vapor cycle. Heating can be achieved by closing the nozzle that is part of the turbine (11) and thereby expanding the air less efficiently than it has been compressed before.

- The reduction of the water content is performed by a water extractor (9) at the coldest point of the whole cycle. To prevent icing of the turbine (11), a reheater is used (10). On high altitudes (or in dry weather), the dehumidification is not needed and can be bypassed (8).

The heat sink for this thermodynamic process is the ram-air flow. This is either driven by the pressure of the ram-air door (12) in flight or actively propelled by fan (14) on ground.

For the sake of clarity, the control elements have been removed from this diagram. Even the prototypical control design for such a plant model with its nonlinearity and reconfigurations is far from trivial. It will be quickly addressed in Section 4 but is essentially out of the scope of this paper. For the moment, let us focus on the interactions between the pack and the overall energy management.

\subsection{Interaction between the Energy Management and the EVCP Pack.}

Being the largest consumer of electrical power (and the second largest of overall power), the EVCP Pack offers substantial optimization for load management.

In case of overload scenarios (for instance when the airplane is operating with a failed generator), the Energy Management Algorithm may choose to reduce the power demand of the pack. To achieve such a desired demand reduction, the energy management manipulates the set points for the EVCP control and of the cabin pressure control. It may

- $\quad$ reduce the amount of fresh air supplied

- increase or lower the pack discharge temperature

- $\quad$ reduce the cabin pressure

How effective each of these actions will be with respect to load reduction strongly depends on the current flight and environmental conditions. Also each of these actions will (slightly or even severely) lower the passenger comfort. The energy management algorithm tries to maximize the lowering in power demand while it tries to minimize the lowering of passenger comfort. It does so based on a simplified model of the aircraft ECS system built from firstprinciples. The first-principle approach has the advantage that it is independent of the detailed technical realization of the EVCP Pack and also more robust in case of local failures or other forms of degradation.

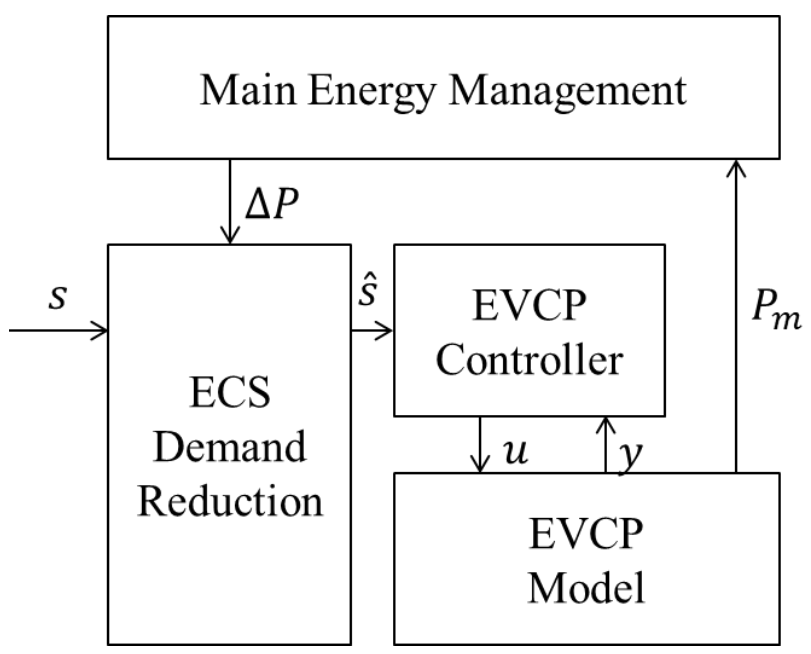

Figure 2: The main energy management issues a power reduction $\Delta P$ which is causing the ECS Demand Reduction to modify the set-point $s$ of the EVCP controller to $\hat{s}$. The actual reaction in terms of power $P_{m}$ is then fed back to the Main Energy Management.

However, this implies that the effective reaction of the EVCP Pack with its in-built controller may deviate from the expectations of the energy management algorithm. The energy management does take this into account by measuring the actual power demand of the pack and making it part of the control loop. Depending on the packs reaction, the energy management algorithm may hence strengthen or weaken its actions.

Understanding this interaction (see Figure 2) between energy management and pack is hence vital and as long as such packs are not available for testing, the test has to be performed virtually.

\subsection{The Main Challenge}

This paper focusses on setting up the EVCP pack model as hard real-time model so that it can be coupled with the inputs and outputs of the energy management algorithm running on a microcontroller.

This is a challenging task because such complicated models typically involve large non-linear equation systems and stiffness. Both prevent hard real time applications. The iterative solution of non-linear equation systems may fail to reach convergence within a fixed time frame. The stiffness of the system often demands stiff-stable ODE solvers which are implicit solvers and share the above problem of indefiniteness in finding a solution.

Fortunately, we can build on prior work (Zimmer, 2019) that is using a new approach for modeling fluid streams. This new approach avoids non-linear equations systems and provides means to manipulate eigendynamics of the mass flow rates without changing its major thermodynamic properties. 


\section{New Methods for Modeling Streams of Thermofluids}

In 2018, we proposed a different format to organize the equations for thermodynamic fluid streams in an object-oriented way (Zimmer 2018, 2020) and implemented this in a Modelica Library. This has been further applied in (Zimmer 2019) and (Otter 2019). The reader is advised to study these papers to gain more insight. Yet we repeat the essential idea and its implications in this section.

\subsection{Fundamentals}

The new approach centers on the decomposition of pressure $p$ into:

$$
p=\hat{p}+r
$$

The term $r$ is thereby defined as inertial pressure:

$$
\Delta r=-L \frac{d \dot{m}}{d t}
$$

Where $L$ is the inertance of a fluid and follows straight from the geometry of the one-dimensional flow and (more importantly) is independent from the thermodynamic state of the fluid:

$$
L=\int \frac{1}{A} d s
$$

where $\mathrm{s}$ is the length in flow direction and $\mathrm{A}$ is the cross section area. This means that given all values of $\hat{p}$, and the current state of mass flow rates $\dot{m}$, we can compute $d \dot{m} / d t$ and all values of $r$ in an arbitrary system by a linear equation system.

Now, what about $\hat{p}$ ? It is denoted as steady massflow pressure because evidently $p=\hat{p}$ for $d \dot{m} / d t=0$. It is possible to compute all $\hat{p}$ for noncyclical flows in explicit form from source to sink if we are willing to assume that the thermodynamic properties are a function of steady-mass flow pressure $\hat{p}$ and not of $p$.

This approximation can be justified for a wide array of applications since:

- it is only a transient phenomena, the error at steady-state will be zero.

- for gases, $r$ is typically very small

- for liquids, the thermodynamic properties are often insensitive to $r$

- many formulas assume steady flow conditions anyway.

Cyclical flows have to be torn-apart by volume elements. Here the volume inlet represents structurally a sink and the outlet a source. If applied correctly the Block-Lower Triangular (BLT) form of complete thermofluid system will have the structure depicted in Figure 3.

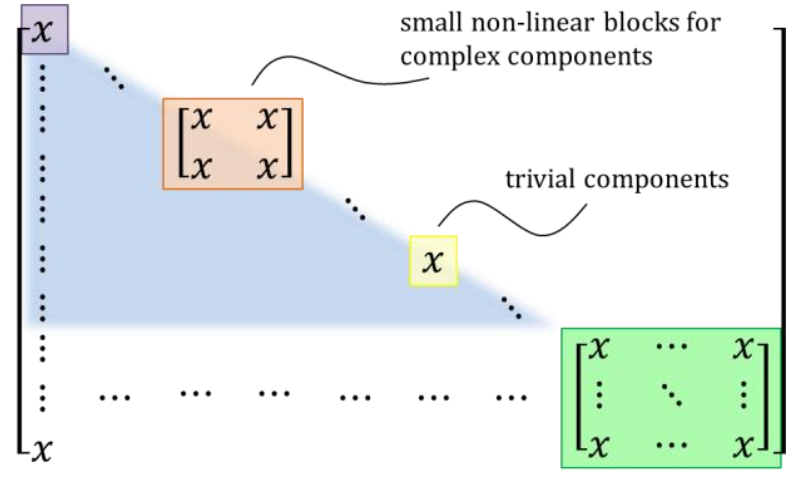

Figure 3: Illustration of the BLT Stucture using the new approach, taken out of (Zimmer, 2018). Green are linear equations. Everything else is potentially non-linear.

\subsection{Implications for Real-time Systems}

The BLT structure already reveals one major implication for real-time system: all non-linear equations form an explicit downstream computation if all individual components are put into explicit form for downstream direction. This is a feasible task and means that no non-linear equation system has to be solved at run-time by an iterative method.

What can we say about the stiffness of the resulting system? Is it possible to apply an explicit solver, like Runge-Kutta 3?

In general, this is not practically feasible. Using natural parameters, the mass-flow dynamics are often very fast with corresponding eigenvalues being strongly negative. The application of explicit solvers would thus demand for an excessively small step width and prevent real-time performance.

The goal is hence to manipulate these mass-flow dynamics so that a sufficiently large step-width can be applied but without impacting the remaining thermodynamic behavior of the system.

Fortunately the mass flow dynamics is largely subject to the linear equation system that is independent of the thermodynamic state and composed out of the law for the inertial pressure. Typically the corresponding matrix $M$ (gree in Figure 3 ) consists in integer coefficients and values for the inertance. We now have three options to manipulate the fast massflow dynamics:

1. Manipulate the values for the inertance in $M$

2. Introduce artificial terms at the 0 -entries of $M$

3. Restructure the matrix by using alternative equations.

It depends on the concrete application which of these options is best to apply. The paper (Zimmer, 2019) outlined the choice for a simplistic bleed-driven environmental control system. Let us repeat this exercise in the following section. 


\section{Mass Flow Dynamics in the EVCP}

Despite the complexity of the pack, there are actually only 4 types of mass-flow dynamics of interest. These are:

- the mass flow dynamics within the ram-air channel,

- the mass flow dynamics of the refrigerant in the vapor cycle model,

- the mass flow dynamics of the main fresh-air ducting,

- The mass flow dynamics of the two by-passes.

\subsection{The Ram-Air Flow}

The control of the ram-air is actually an intricate issue. It is either controlled by a fan and/or by the ram-air door. The latter is thereby highly non-linear dependent on the current flight conditions especially the true air speed.

Fortunately, none of this is of concern. Simply manipulating the inertance of the ram-air flow does the trick (Method 1). Applying a factor of 50 is large enough to suppress too fast dynamics and yet the flow remains nicely controllable.

A solution in line with the next two sections could be designed as well but it was not necessary.

\subsection{The Refrigerant Flow}

The compressor of the vapour cycle (15) is power controlled. Typically it adapts the pressure raise for the current mass flow rate of refrigerant. So the tuple of power $P$ and mass-flow rate $\dot{m}$ determines the pressure raise $\Delta p$

$$
(P, \dot{m}) \rightarrow \Delta p \rightarrow\left(\frac{d \dot{m}}{d t}, r\right)
$$

where $\dot{m}$ is a state whose derivative is dependent on $\Delta p$. Typically the time constant is very fast.

For a real-time solution, we hence choose to restructure the equation system (Method 3 ). Now we define the pressure raise as a state and prescribe the mass-flow rate such that the desired power level is reached. Its derivative and the corresponding inertial pressure directly follow from this.

$$
(P, \Delta p) \rightarrow\left(\dot{\mathrm{m}}, \frac{d \dot{m}}{d t}, r\right) \rightarrow\left(\frac{\Delta p}{d t}\right)
$$

We determine the derivative of $\Delta p$ by stating

$$
\frac{\Delta p}{d t} \tau=r
$$

We can now nicely control the time constant $\tau$ of the pressure raise and avoid too fast dynamics. This technique has also previously been outlined for valves in (Zimmer, 2019).

\subsection{The Fresh-air Flow}

The main compressor (5) shall control the mass flow to the desired set-point $\dot{m}_{\text {set }}$. As such it is even more straight forward than the vapor cycle compressor. We can restructure the dependencies straight forward to

$$
\left(\dot{m}_{s e t}\right) \rightarrow\left(\dot{\mathrm{m}}, \frac{d \dot{m}}{d t}, r\right) \rightarrow\left(\frac{\Delta p}{d t}\right)
$$

and again apply

$$
\frac{\Delta p}{d t} \tau=r
$$

to keep the dynamics in check

\subsection{The By-pass Flows}

The natural dynamics of opening and closing by-pass valves is highly nonlinear. Mostly this is because it not only involves flow control valves but also check-valves to prevent potential counter flow. Check-valves are very non-linear (ideally even discrete) in their behavior. In this application, we can however avoid these troubles by simply stipulating the mass-flow split in the splitter (2) and (8).

In a classic free splitter (A being inlet, $\mathrm{B}$ and $\mathrm{C}$ being outlet), the mass flow balance is upheld and all inertial pressures are set equal:

$$
\begin{gathered}
m_{A}+m_{B}+m_{C}=0 \\
r_{B}=r_{A} \\
r_{C}=r_{A}
\end{gathered}
$$

If we choose to prescribe the mass flow split by a parameter $0<s<1$, we gain one constraint equation and in turn have to remove the equality of all inertial pressures:

$$
\begin{gathered}
m_{A}+m_{B}+m_{C}=0 \\
s \cdot m_{A}=-m_{B} \\
r_{A}=s r_{B}+(1-s) r_{C}
\end{gathered}
$$

For the last equation, we assume that the flow is split under equal density. Then the forces acting on the corresponding cross-section areas of both sides have to be equal to each other (illustrated in Figure 4). This implicates the additional assumption that kinetic energy needed to change the mass flow rate can be exchanged between the two bypasses.

Such an assumption can be made (to a limited degree) for bypasses where one flow also propels its 
bypass flow such as in a dedicated gap in a heat exchanger or the surrounding of a fan. For the bypasses as in (13) or (14) this assumption is actually invalid.

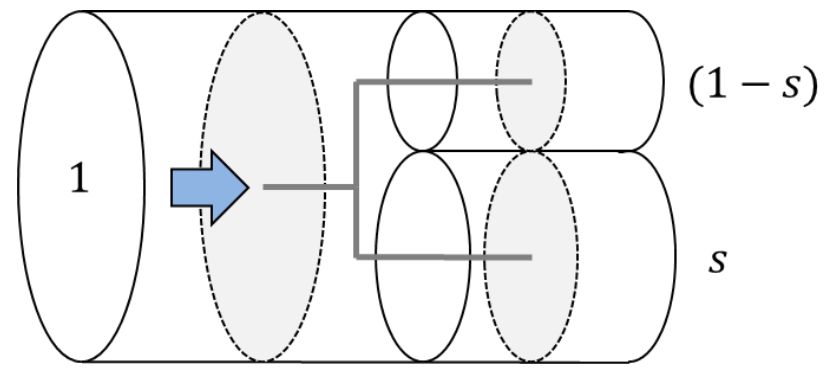

Figure 4: Illustration of a splitter with an enforced flow regime. A corresponding thought experiment illustrates a mechanic construction by 3 rigidly connected discs. The inertial pressures on these discs have to match resulting in the corresponding equation.

Fortunately we can still apply this method here because the bypass is either fully open or fully closed. At these extremes, this yields valid results and at the moment, the transient behavior is of no major interest.

A word of warning: for bypasses that are really meant to regulate something by controlled mixing (like bypasses for a high-frequency temperature control or trim-air valves), this method should definitely not be applied.

For the moment, we are seeing this solution as an intermediate solution to be replaced with better alternatives in the future.

\section{Control of the EVCP}

To reach the desired set-point (in terms of temperature, mass flow and maximum water content) a prototypical controller needs to be developed. Furthermore, a decision strategy for the opening and closing of the bypasses is needed.

All this is far from trivial and exceeds the scope of this paper. However, for the general understanding we briefly outline how a prototypic controller for the $\mathrm{EVCP}$ can be designed in functional terms.

\subsection{Fresh Air Flow Control}

The control of the desired amount of fresh air is performed by powering the main compressor (5). The main compressor may be supported by the base compressor. The decision whether to switch on or off the support the bypass is done based on the pressure ratio between air inlet pressure (1) and main compressor outlet pressure (5). The switch is implemented using hysteresis. This pressure ratio may also be influenced by the bypass to the turbine (8).

\subsection{Temperature Control}

For the cooling case, the pack discharge temperature is controlled by the flow of ram-air which is in turn controlled by the ram-air door or subsequently (at or near) ground by the ram-air fan. The gain factor of the control inputs is thereby highly non-linearly dependent on the flight conditions. Temperature control is also relatively slow due to the heat capacity of the heatexchangers and the latency of the refrigerant in the vapour cycle.

For the heating case, the ram-air flow needs to be limited to a minimum and the variable nozzle of the turbine (11) lowers its efficiency. The inefficient expansion of previously compressed air enables heating.

\subsection{Humidity control}

The effectiveness of water separation is essentially influenced by the temperature at the water separator (9). In combination with the overall pack discharge temperature control this is influenced by the variable nozzle of the turbine (11). An inefficient expansion requires a lower temperature at the inlet of the reheater (10) at hence enables a more efficient water extraction.

In case the fresh air has too little water content in the first place, the energy expensive water extraction can be bypassed (8) unless heating is required. Also this discrete decision is implemented by hysteresis.

Humidity control only extracts water. Water is not added in case the air is too dry (unfortunately).

\subsection{Remaining Degrees of Freedom.}

There remain two degrees of freedom that can be used to optimize the performance during operation as part of the overall thermal management.

One is the powering of the compressor of the vapour cycle (that again contains its own sub-controllers and potential bypasses). This can be used to regulate the temperature gradient in the ram-air channel so that each heat-exchanger is used effectively.

The other degree of freedom is the turbine pressure ratio. This enables a trade-off between electric power needed by the EVCP Pack (high pressure ratio) or more additional drag caused by the ram-air channel opening (low pressure ratio).

In summary the overall control implementation embraces tasks from high-level thermal management down to internal pack reconfiguration and is hence a very complex task. 


\section{Simulation Results}

The final model including the controller includes 44 states and more than 1500 time varying variables.

A few of the states are needed for the design of the controllers but most of them describe time-constants of the heat-exchangers and some of them have been introduced in order to enable an explicit computation of the non-linear equations within a component (Zimmer, 2013).

After the manipulations (as sketched in Section 3), the dynamics of all states is slow enough to enable a real-time simulation. Runge-Kutta 3 with a step-width of $0.1 \mathrm{~s}$ was chosen as integration method.

With these settings, the model performs 5 times faster than real-time on a conventional desktop PC including writing the simulation results on the hard disk. The computation for the vapour cycle thereby dominates the computational demand (>85\%). This is because of the complex media model and because of the complex moving boundary heat-exchanger models.

Figure 5 shows the simulation result for a simplistic ascent of the airplane from ground to flight altitude. This scenario changes the environmental conditions, the cabin pressure requirements and the required packdischarge temperature. Some of these transients are relatively quick.

The three curves in Figure 5 show the pack discharge temperature by 3 different curves: the desired set point (the control target) and the actual resulting pack discharge temperature from the original model and the modified real-time model. The occurring differences demand explanation.

For both models there is a noticeable difference between the set-point and actual value indicating a relatively poor control quality. There are good reasons for that: First of all, the implemented controller is prototypical and certainly not optimal. Second the opening and closing of bypasses that happens during ascent, causes major disturbances that are difficult to compensate without preemptive action. Last but not least, the control authority is limited also for a real system. It simply takes time to react. Hence the actual system would also include further bypasses for higherfrequency temperature control. These are omitted here because we currently focus on the main energetic behavior and not on comfort. However, the gain of control authority by dedicated temperature control valves has its energetic penalty as well and hence they will be included in future versions of our work.

The difference between the original model and its real-time derivative is essentially due to the disturbance on the control due to the reconfigurations: turbine bypass opens around $33 \mathrm{~min}$, compressor bypass closes around $50 \mathrm{~min}$. As outlined in Section 3.4 , the quality of the bypass regulation is changing during the transition period and consequently also the quality of the disturbance. Hence the two models (who share their control scheme) react differently.

For the moment, this difference between the models is acceptable for us because the general model uncertainty (also regarding the control design) with respect to reconfigurations is very high. However, this is something that requires more effort in the future and is currently regarded as an intermediate solution.

Also these results indicate that such reconfigurations may pose difficulties in interaction with an energy management and hence a closer investigation of this topic may be needed.

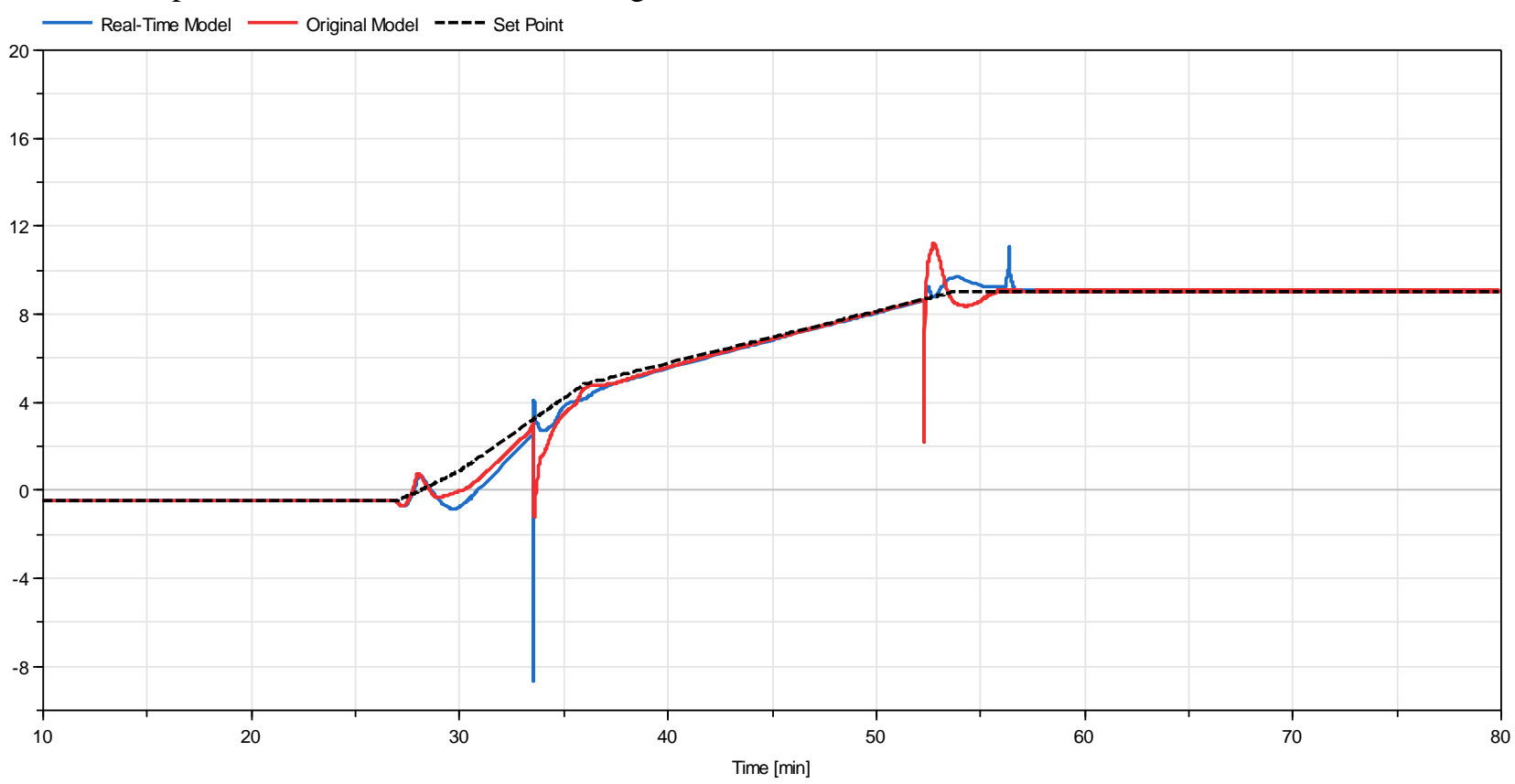

Figure 5: Pack discharge temperatures at flight ascent. This graph shows the control quality w.r.t to the setpoint and the difference between the real-time simulation and the original model. 


\section{Conclusions}

\subsection{Summary}

The provided example of an EVCP successfully demonstrates that hard real-time simulation of even complex thermofluid systems becomes a fairly well achievable task with Modelica. The example contains almost all what is typically regarded as challenging: vapor cycles (see also Schulze, 2011), complex heatexchanger models, bypasses, compressors, turbines and complex boundary conditions. Also it handles 3 different kinds of media: moist air, water, and a refrigerant. This gives us confidence for future challenges and larger systems.

The main remaining challenge is a better solution for bypasses with check-valves. The proposed solution here is good enough as long as the transition period is not important but the reactions of the control system reveal noticeable differences after all.

Finally, the hard real-time simulation of such thermodynamic processes should not be such an issue in the first place. Typically all time-constants of interest are well above the desired communication interval. Only large non-linear equation system and fast mass-flow dynamics oppose a quick simulation. The new approach (Zimmer, 2018, 2019), (Otter, 2019) based on the inertial pressure allows to prevent the former and isolate the latter.

A final remark (because it often gets forgotten): Hard real-time simulation is actually a very slow form of simulation because it solely focusses on a guaranteed response time. Without doing all of the work outlined in this paper but simply using implicit solvers with variable step-width such as ESDIRK23 (Jørgensen, 2018) on the original model, the systems simulates much faster than real-time, just not with a guaranteed response time.

\subsection{Outlook on future work}

This paper focused on setting up the main EVCP model for hard real-time simulation. The main task, which is the coupling with overall management algorithm, has yet to be conducted.

This will reveal how well the energetic behavior of a complex system such as the EVCP can be handled by an overall energy management. Another questions is how sudden events such as the reconfiguration of the system impact the energy management in return.

\section{Acknowledgements}

The work is receiving funding from European Union's Horizon 2020 for the Clean Sky 2 Joint Technology Initiative under grant agreement H2020-CS2-CFP032016-01 GAN 737792.

\section{References}

Bender, Daniel (2018) Exergy-Based Analysis of Aircraft Environmental Control Systems and its Integration into Model-Based Design. Dissertation, Technische Universität Berlin.

Steffen Golle et. Al., (2016), Betriebsphasenabhängig steuerbare Flugzeugklimaanlage und Verfahren zum Betreiben einer derartigen Flugzeugklimaanlage, Patent, DE102015207447A1, 27.10.2016

John Bagterp Jørgensen, Morten Rode Kristensen, Per Grove Thomsen (2018) A Family of ESDIRK Integration Methods In: Numerical Analysis

Otter, Martin et al., (2019) Thermodynamic Property and Fluid Modeling with Modern Programming Language Constructs. In: Proceedings of 8th International Modelica Conference. 13th International Modelica Conference, Mar 04-06, 2019, Regensburg, Germany.

Schulze, Christian (2011) Real-Time Simulation of Vapour Compression Cycles. In: Proceedings of 8th International Modelica Conference., 20.-22. Mar 2011, Dresden.

Zimmer, Dirk (2020) Accepted for publication: Robust Object-Oriented Formulation of Directed Thermofluid Stream Networks In: MCMDS Journal.

Zimmer, Dirk (2019) Towards Hard Real-Time Simulation of Complex Fluid Networks. In: Proceedings of the 13th International Modelica Conference, 04.-06. Mär. 2019, Regensburg, Deutschland

Zimmer, Dirk and D. Bender, A. Pollok (2018) Robust Modeling of Directed Thermofluid Flows in Complex Networks. In: Proceedings of the 2nd Japanese Modelica Conference, Tokyo, Japan

Zimmer, Dirk, (2013), Using Artificial States in Modeling Dynamic Systems: Turning Malpractice into Good Practice. In: Proceedings of the 5th International Workshop on Equation-Based Object-Oriented Languages and Tools (EOOLT), Nottingham, United Kingdom 